\title{
Touching Motion: rTMS on the Human Middle Temporal Complex Interferes with Tactile Speed Perception
}

\author{
Demis Basso • Andrea Pavan · Emiliano Ricciardi • \\ Sabrina Fagioli · Tomaso Vecchi · Carlo Miniussi • \\ Pietro Pietrini
}

Received: 8 August 2011 / Accepted: 13 February 2012/Published online: 25 February 2012

(C) Springer Science+Business Media, LLC 2012

\begin{abstract}
Brain functional and psychophysical studies have clearly demonstrated that visual motion perception relies on the activity of the middle temporal complex (hMT+). However, recent studies have shown that hMT + seems to be also activated during tactile motion perception, suggesting that this visual extrastriate area is involved in the processing and integration of motion, irrespective of the sensorial modality. In the present study, we used repetitive transcranial magnetic stimulation (rTMS) to assess whether hMT+ plays a causal role in tactile motion processing. Blindfolded participants detected changes in the speed of a grid of tactile moving points with their finger (i.e. tactile modality). The experiment included three different conditions: a control condition with no TMS and two TMS conditions, i.e. hMT+-rTMS and posterior parietal cortex (PPC)-rTMS. Accuracies were
\end{abstract}

D. Basso $(\bowtie)$

Faculty of Education, Free University of Bozen-Bolzano, Viale Ratisbona, 16, 39042 Bressanone, BZ, Italy

e-mail: demis.basso@unibz.it

D. Basso

Centro di Neuroscienze Cognitive Applicate, Rome, Italy

A. Pavan

Cognitive Neuroscience Sector, SISSA, Trieste, Italy

E. Ricciardi · P. Pietrini

Department of Experimental Pathology, BMIE,

University of Pisa, Pisa, Italy

S. Fagioli

Department of Clinical and Behavioral Neurology and Memory

Clinic, IRCCS Santa Lucia Foundation, Rome, Italy

T. Vecchi

Cognition Psychology Neuroscience Lab, University of Pavia,

Pavia, Italy significantly impaired during hMT+-rTMS but not in the other two conditions (No-rTMS or PPC-rTMS), moreover, thresholds for detecting speed changes were significantly higher in the hMT+-rTMS with respect to the control TMS conditions. These findings provide stronger evidence that the activity of the hMT+ area is involved in tactile speed processing, which may be consistent with the hypothesis of a supramodal role for that cortical region in motion processing.

Keywords Repetitive TMS - Speed detection - Tactile · Temporal lobe

Introduction

It has been widely demonstrated that the perception of visual motion engages the human extrastriate cortical region, i.e. the middle temporal complex (hMT+) (Born

\author{
T. Vecchi \\ Brain Connectivity Center, IRCCS Mondino, Pavia, Italy \\ C. Miniussi \\ Department of Biomedical Sciences and Biotechnologies, \\ National Institute of Neuroscience, University of Brescia, \\ Brescia, Italy \\ C. Miniussi \\ Cognitive Neuroscience Section, IRCCS San Giovanni di Dio \\ Fatebenefratelli, Brescia, Italy
}


and Bradley 2005; Tootell et al. 1995). However, it has also been suggested (Hagen et al. 2002; Blake et al. 2004) that the extrastriate cortex, along the earliest regions of the dorsal pathway, can process information independently from sensorial modality. This view was recently supported by a number of brain functional (Matteau et al. 2010; Ptito et al. 2008; Beauchamp et al. 2007) and psychophysical (Konkle et al. 2009; Bicchi et al. 2008) studies, providing support for the involvement of the hMT + in non visual (i.e. tactile and aural) motion perception. For example, Konkle et al. (2009) demonstrated a bidirectional relationship between visual and tactile motion processing. In particular, using the motion adaptation paradigm, they examined transfer of the motion aftereffect (MAE), where prolonged exposure to directional motion causes a subsequently presented stationary or counterphase (flicker) stimulus to be perceived as moving in the opposite direction (for a review, see Mather et al. 2008). Interestingly, a reliable MAE was found when adapting to visual motion and testing with tactile motion, and vice versa. This cross-modal aftereffect provides evidence that the processing of visual and tactile motion relies on shared representations that may play an important role in modality-specific perception.

Accordingly, in an fMRI study, Ricciardi et al. (2007) showed that, in both sighted and congenitally blind participants, the hMT+ was activated during two tasks involving passive perception of visual and/or tactile moving stimuli. These results suggest that visual perception is not a necessary prerequisite in order to activate hMT+. However, several studies postulated a functional equivalence between imagery and perceptual processes (e.g. Kosslyn 1994 for a review) as well as a shared neural network (Kosslyn et al. 2001), although differences are also reported (Ganis and Schendan 2008) specifically showing that the same neural population may be differentially activated during imagery versus perceptual processes. Further, different studies indicated that visual imagery may be involved in visual perception in sighted individuals (e.g. Lacey et al. 2010) through top-down processes (Bar 2007; Lacey et al. 2009). Taken together, these results indicate a complex functional structure involving both perceptual and imagery processes and confirmed the presence of a supramodal organisation of the dorsal stream (Beauchamp 2005). Although convergent, the results obtained from these studies involved correlative techniques (i.e. PET and fMRI) and thus, suffer from the criticism that these imaging data are only correlated to the behavioural results (Van Orden and Paap 1997). The transcranial magnetic stimulation (TMS) method allows researchers to investigate causality in the brain-behaviour relationship, by temporarily affecting the activity of neurons in brain areas subjected to a magnetic field administered by a coil positioned over the scalp. One advantage of TMS over other brain functional methodologies is that TMS can be used to demonstrate that a specific brain region is causally activated while performing a given task (Walsh and PascualLeone 2003). In previous work, TMS was used mainly to assess visual motion perception in the hMT+ (McKeefry et al. 2008; Ruzzoli et al. 2010; Sack et al. 2006; Théoret et al. 2002), but the role of this area in non-visual motion perception, such as with tactile dynamic stimuli, has only been explored previously in a study by our group (Ricciardi et al. 2011). Participants were asked to press a key whenever they detected a change of speed in a translational Braille-like surface on a cylinder that rotated continuously at five different speeds. Seven pulses of repetitive TMS (rTMS) at $10 \mathrm{~Hz}$ were applied after each transition, over either left hMT + or a control site corresponding to the interhemispheric sulcus. The results showed increased reaction times and decreased accuracy only when the stimulation was applied to the left hMT+, but not to the control site, and the TMS effect was higher when participants had to detect speeds with a small difference between them rather than with a larger difference. These results allowed us to conclude that the hMT + area is also involved in tactile motion perception.

In the present study we aimed at replicating and extending the study by Ricciardi et al. (2011), by including additional experimental conditions and analyses, since several methodological limits of the previous experiment may have hindered interpretation of the main result. First of all, Ricciardi et al. (2011) did not test some crucial parameters of the experimental task such as different sets of speeds, preventing the possibility to get additional information on perceptual processing. Therefore, we devised a preliminary experiment, in which three sets of speeds were tested to determine whether the parameters used in our previous study (Ricciardi et al. 2011) were the most appropriate. Although, we expected to obtain confirmation for the parameters used in the previous study, other sets of speeds (with closer distances between them) were also tested. The rationale was that, if participants could detect speeds changes from a more precise set of speeds, then we could (i) obtain enhanced perturbation of the performance (since rTMS effects are measured on threshold shifts), and (ii) determine the psychophysical limits of the tactile motion detection process.

In the main experiment, the most appropriate parameters obtained from the preliminary experiment were assessed, while rTMS was applied either over the hMT + or a control site (i.e. the interhemispheric sulcus in the posterior parietal cortex, PPC). We used the same procedure and stimulation sites as Ricciardi et al. (2011), in order to add reliability to their results. In addition, we included a supplementary control condition without stimulation (NoTMS), which was not present in the earlier study of 
Ricciardi et al. (2011). Critically, the No-TMS condition would allow better disentanglement of rTMS-mediated effects, paying particular attention to the relationship between the neural activity induced by TMS and the resulting task execution (Miniussi et al. 2010; Ruzzoli et al. 2010). In particular, this condition allowed us to verify whether TMS stimulation of the PPC produced an improvement/decline in performance, or no difference. In the latter case, the no-stimulation condition would represent a better control condition. This measure was necessary to quantify any non-specific TMS effects on task performance.

Moreover, in the present study we sought to estimate detection thresholds, since they represent a more direct measure of the effect of the TMS. Thus, we assessed whether rTMS affects the speed change detection (Ricciardi et al. 2011) influencing: (i) speed change detection thresholds, while keeping the slopes of the psychometric functions constant, or (ii) slopes, while maintaining the detection thresholds constant, or (iii) both parameters (Harris et al. 2008; Ruzzoli et al. 2010). In the first case, variation of the detection thresholds could be fully attributed to interference of the process performed in the stimulated region. In the second case, the variation would merely produce a change in the strength of the effective signal processing, due to a greater internal noise thus rendering the task harder and affecting the ability of participants to detect speed changes (Buss et al. 2009; Mather and Pavan 2009). Finally, if both these parameters changed, then both of the processes could account for the change in performance.

Therefore, our operational aims were: (i) to specify the set of speeds that produce a reliable performance in detecting speed changes more effectively, and (ii) to define the characteristics of the underlying mechanism/s involved in tactile motion perception, in terms of detection thresholds and slopes (i.e. trying to disrupt these parameters with the rTMS stimulation). The differences in both paradigm and data analysis seek to improve the entire methodological setting to assess reliably whether hMT+ is involved in tactile motion perception.

\section{Preliminary Experiment}

The aim of the preliminary experiment was to determine the most effective parameters of the behavioural task, thus improving the procedure used by Ricciardi et al. (2011). In fact, that study omits testing of different sets of speeds. Therefore, in the present experiment, three sets (determined by the diameter of a set of cylinders) were tested, in order to obtain a range of data suitable to evaluate the reliability of the participants' performance.

\section{Method}

\section{Participants}

Twelve subjects (4 males; mean age: 24 years; age range, 21-30 years), recruited through advertisements, participated in the pilot experiment. All subjects were righthanded, according to the Edinburgh Handedness Inventory (Oldfield 1971). All participants were free from any medical, neurological or psychiatric disorder, and were not taking any medication. All gave their written informed consent after the study procedures had been explained. The whole experimental protocol was approved by the Ethics Committee of the Department of Psychology, University of Pavia (Italy).

\section{Apparatus and Procedure}

Three cylinders with a surface made of a regular grid of tactile points (diameter: $0.5 \mathrm{~mm}$, height: $0.5 \mathrm{~mm}$, density: $0.8 / \mathrm{cm}^{2}$; dot spacing is displayed in Fig. 1) were used. The small, medium and large cylinders were 5.1, 6.9 and $10.0 \mathrm{~cm}$ in diameter, respectively. The cylinders were rotated outwardly at five different speeds by an electric engine connected to a computer through a controller. This received impulses from a PC parallel port and translated them into variations of the power passed to the engine; speeds changed instantaneously. Grids of tactile points, the larger cylinder and the speeds produced by the engine were the same as those of Ricciardi et al. (2011). The five speeds were set to increase regularly from 0.42 to 0.62 revolutions per second, whereas the tangential speeds were proportional to the diameter of each cylinder. The speeds for the smaller cylinder were $8.29,8.78,9.20,9.71$, and $10.15 \mathrm{~cm} /$ $\mathrm{s}$, the speeds for the medium cylinder were $10.99,11.63$, $12.19,12.87$, and $13.45 \mathrm{~cm} / \mathrm{s}$ and the speeds for the largest

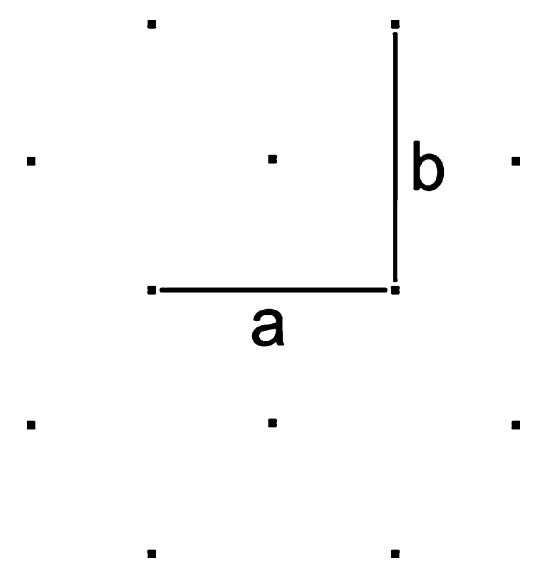

Fig. 1 Dot spacing of the surface of the cylinders: $0.85 \mathrm{~cm}$ for the direction parallel (a) and $0.80 \mathrm{~cm}$ for the direction perpendicular to the cylinder rotation (b) 
cylinder were $16.03,16.96,17.78,18.77$, and $19.62 \mathrm{~cm} / \mathrm{s}$. The five speeds for each cylinder size were coupled to obtain 4 changes in speed. Each speed change was either ascending (i.e. passing from a slower to a faster speed) or descending (from a faster to a slower speed). A sharp transition was always used to change from one speed to the other.

The arrangement of the speed changes in pairs led to a total of 20 different combinations, separated into four levels on the basis of the distance between the two speeds, ranging from a distance of 1 (e.g. speeds 3-4) to a distance of 4 (e.g. speeds 1-5). For sake of clarity, we will refer to the distance between two speeds as the "speed gap". Because four pairs of speeds were available for the smallest speed gap (i.e. a speed gap of 1 could originate from speeds $1-2$, speeds $2-3$, speeds $3-4$, and speeds $4-5$ ), but only one pair of speeds was available with the higher speed gap (speeds 1-5), the pairs for larger speed gaps were repeated to obtain the same number of stimuli for each category (i.e. eight, when considering the ascending and descending speed directions). Moreover, eight trials with no change of speed (i.e. a speed gap of 0 ; catch trials) were included in each block, giving a total of forty trials $(32+8)$. These were presented through concatenation of the pairs of speeds into a single flow (lasting approximately $5 \mathrm{~min}$ ); speeds were presented consecutively, with the transitions between speeds balanced to measure each change size equally. ${ }^{1}$ The subjects' task consisted of pressing the spacebar whenever a change in the speed of the surface was detected. The inter-change time was varied from 4 up to $6 \mathrm{~s}$, to avoid rhythmic responses by participants; this range allowed sufficient time for the participants to perceive the surface motion and to execute a response.

The cylinder-engine system was hidden in a polystyrene box, with an aperture on the upper part to allow participants to insert the stimulated finger while comfortably supporting the other fingers. Participants touched the cylinder with either the index or the middle finger of the dominant hand (alternated, between blocks). Participants were administered one learning block (to familiarise them with the apparatus/stimuli and task) and six experimental blocks, obtained from the combination of the three cylinders of different sizes with the two fingers. The presentation order was randomised between subjects. Participants were blindfolded while performing the task and pink noise at 50 decibels was used to mask the engine's changes of speed. All responses within a trial, subsequent to the initial

\footnotetext{
${ }^{1}$ For example: Considering the largest cylinder, an initial speed of $16.96 \mathrm{~cm} / \mathrm{s}$, which was maintained for $4.5 \mathrm{~s}$, then changed to a speed of $19.62 \mathrm{~cm} / \mathrm{s}$ (speed gap $=3$, ascending direction), maintained for $4 \mathrm{~s}$. The speed was then changed to a speed of $17.78 \mathrm{~cm} / \mathrm{s}$ (second trial, with speed gap $=2$ and descending direction) that was maintained for $5.5 \mathrm{~s}$, and so on.
}

response, were considered false alarms. Moreover, in order to detect false alarms within the first key-presses, these were controlled using a two-step method: in the first step, a response was excluded if its RT was lower than $350 \mathrm{~ms}$ or higher than 4,000 ms, in the second, if its RT was outside two standard deviations, calculated individually for each participant. After these steps, the number of excluded responses was 53: that is, a mean of 0.67 FAs per block/ participant.

Trial presentation, consisting of the transmission of the impulses through the PC parallel port, was controlled with Presentation software (Neurobehavioural System Inc., Albany, CA, USA).

A series of repeated-measure ANOVAs was performed on the percentage of correct responses, separately for each cylinder, since the three cylinders produced a different pattern of speeds. The following independent within-subjects variables were compared: Speed Gap (four levels), Speed Direction (two levels: ascending or descending) and Finger (two levels: index or middle). Because the level speed gap $=0$ was a repetition of the previously presented speed, it had no direction; therefore, it was excluded from the ANOVA. Post hoc pairwise comparisons were performed with Bonferroni correction, assuming an alpha level of 0.05 .

\section{Results and Discussion}

Two participants did not produce responses for any cylinder and were excluded from the analysis. Data from a third participant were not included in the analysis for the largest cylinder, due to an error in the data collection system.

Figure 2 shows the results of the preliminary Experiment. The percentage of times reporting a speed change obtained with the large cylinder ranged from 39.7\% (smaller gap) to $78.7 \%$ (larger gap) and was generally higher than that obtained with the small and medium cylinders across all speed gaps tested. Indeed, the percentage of times reporting a speed change for the small and medium cylinders ranged from $17.1 \%$ (smaller gap) to $32.0 \%$ (larger gap) and from $33.2 \%$ (smaller gap) to $65.3 \%$ (larger gap), respectively.

For the largest cylinder (Fig. 2, left panel), a repeated measures ANOVA showed a significant effect of the Speed Gap $\left(F_{3,24}=15.82, \quad p<0.01, \quad \eta^{2}=0.66\right)$. Bonferroni corrected pairwise comparisons showed a significant difference between speed gap 1 and speed gap 4 and between speed gap 2 and speed gap 4 . In addition, a significant interaction between Speed Gap and Speed Direction (i.e. ascending or descending $), \quad\left(F_{3,24}=3.25, \quad p<0.05\right.$, $\eta^{2}=0.28$ ) was found. Bonferroni corrected pairwise comparisons for the descending direction revealed a 


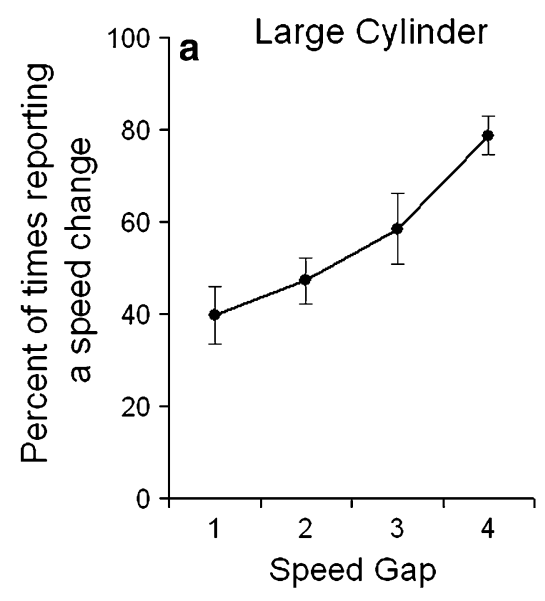

Fig. 2 Results of the preliminary experiment. Because we did not obtain any significant effects of speed direction or finger for the three cylinders, we collapsed the levels of these variables and represented the percentage of times reporting a speed change as a function of the

significant difference between speed gap 1 and speed gap 3 , speed gap 1 and speed gap 4, and speed gap 2 and speed gap 4. For the ascending direction, Bonferroni corrected pairwise comparisons showed a significant difference only between speed gap 2 and speed gap 4 . No other variables or interactions reached significance.

For the medium cylinder (Fig. 2, middle panel), no significant effects were obtained.

For the small cylinder (Fig. 2, right panel), we found a significant effect of the Speed Gap only $\left(F_{3,27}=5.17\right.$, $p<0.01, \eta^{2}=0.36$ ). In addition, no main effects were found for the Finger used (index or middle) across the three cylinders.

Overall, in this preliminary experiment, the highest percentage of times reporting a speed change was obtained only with the larger cylinder across all the speed gaps.

\section{Main Experiment}

The preliminary Experiment revealed that the most reliable performance was obtained using the largest cylinder. In Experiment 2, we used this optimal stimulus configuration to assess whether a transient interaction with the activity of the hMT+ induced by rTMS affected specific processing in the tactile detection of speed changes.

\section{Participants}

Fourteen right-handed subjects (10 males; mean age: 25 years; age range; 20-32 years) were recruited through advertisements. All participants were free from any medical, neurological or psychiatric disorder, had no contraindications to rTMS (Rossi et al. 2009) and were not taking

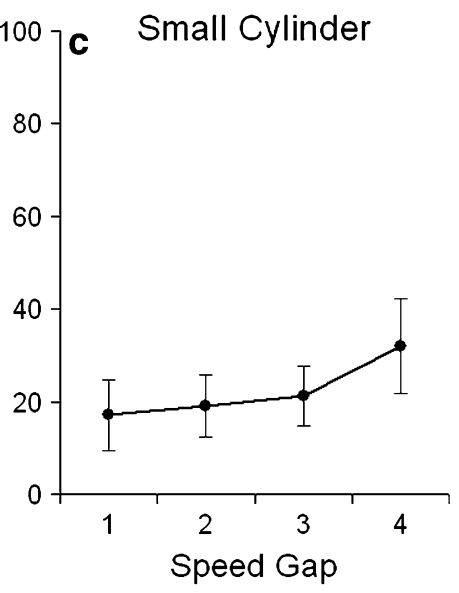

speed gaps. a shows the percentage of times reporting a speed change for the large cylinder, $\mathbf{b}$ for the medium cylinder, and $\mathbf{c}$ for the small cylinder. Error bars \pm SEM

any medication. All participants gave their written informed consent after the study procedures and the risks involved had been explained, and received payment for participating to the study. The whole experimental protocol was approved by the Ethics Committee of IRCCS Fatebenefratelli, Brescia (Italy), according to the Declaration of Helsinki (1964).

\section{Materials and Procedure}

\section{Stimuli and Procedure}

Stimuli and procedure were the same as the preliminary Experiment, except that only the largest cylinder (diameter: $10 \mathrm{~cm}$ ) was used.

Participants were presented with six blocks: two control and four experimental blocks. The between-block factors were the rTMS conditions (three levels: No-rTMS, PPCrTMS, and hMT+-rTMS) and Finger (two levels: index vs. middle, used in alternated sequence). The within-block factors were Speed Gaps (five levels) and Speed Direction (two levels: ascending vs. descending). An additional initial block without TMS was presented to allow participants to familiarise themselves with the apparatus/stimuli and task. To obtain a homogeneous sample, and emphasise the effect of TMS stimulation, participants whose performance in this initial block were below a threshold of $30 \%$ response rate were excluded from further experimental blocks. Participants pressed the spacebar with their left hand whenever they detected a change in speed. To estimate the effectiveness of the stimulation, the following logistic function was fitted to the data (Lam et al. 1996; Gleich et al. 2006): 
$p(x)=a+(b-a) \times\left(1 /\left(1+e^{-((x-m) / s)}\right)\right)$

where $p$ is the probability to report a speed change, $x$ is the stimulus level, $a$ is the false alarm probability, $b$ is the maximum response probability, $\mathrm{m}$ is the midpoint of the function and $s$ is the slope. Post hoc pairwise analyses were performed using the Bonferroni correction with $\alpha=0.05$.

\section{TMS Protocol}

TMS was performed using a Magstim Rapid (The Magstim Company, UK) and a figure-eight coil (double $70 \mathrm{~mm}$ ), which produced biphasic waveform magnetic stimuli with a pulse width of $0.2 \mathrm{~ms}$. Stimulation sites were the hMT+ area (experimental site), identified at the Talairach coordinates $49,-62,5$ (the location of peak activation observed in the study of Ricciardi et al. 2006) and an interhemispheric location in the PPC (control site) at the coordinates $0,-76,30$. The sites were identified on the subject's scalp using the SofTaxic Evolution Navigator system (E.M.S., Bologna, Italy). This system allows 3D virtual reconstruction of the cerebral cortex in Talairach coordinates with a mean error of $2.11 \mathrm{~mm}$ and a standard deviation of $2.04 \mathrm{~mm}$, on the basis of digitised skull landmarks as well as 50 additional, uniformly distributed points mapped on the scalp with a graphic user interface and a 3D optical digitiser (NDI, Polaris Vicra).

Stimulation over the experimental site was performed with the handle of the stimulation coil pointing $45^{\circ}$ from the midline. Stimulation over the control site was performed with the handle of the coil parallel to the midline, pointing anteriorly. The stimulus intensities were determined by measuring the individual motor threshold (MT) at rest and defined as the minimum percentage of the stimulator output that evoked a visually detectable twitch in the contralateral hand when contracted (Pridmore et al. 1998). Since the task was not a visual task, we chose TMS intensity based on a standard individual MT procedure. This option was also preferred since the individual phosphene threshold differed considerably between subjects, and is generally higher than the MT. The stimulator output was set at $110 \%$ of the MT for the stimulation of the target sites (mean stimulation: $44 \%$ of the maximum stimulator output; range: 38-52\%). Repetitive TMS consisted of seven TMS pulses at a frequency of $10 \mathrm{~Hz}$, starting $200 \mathrm{~ms}$ after the change of speed; this temporal window was chosen in order to (a) intercept the processing in the $\mathrm{hMT}+$ area (for $600 \mathrm{~ms}$ ), and (b) avoid an overlap between the onset of TMS pulses and speed change (200 ms allows for early processing in the primary sensorial area). These parameters were in accordance with the safety guidelines (Rossi et al. 2009).

\section{Results}

Since a repeated measures ANOVA did not report neither a significant effect of the Speed Direction (i.e. ascending vs. descending $)\left(F_{1,13}=2.56, p>0.05, \eta^{2}=0.17\right)$ nor a significant effect of the Finger $\left(F_{1,13}=0.24, p>0.05\right.$, $\left.\eta^{2}=0.02\right)$, data were collapsed across these two main factors.

An additional repeated-measures ANOVA reported a significant effect of the main factor TMS $\left(F_{2,26}=15.4\right.$, $\left.p<0.05, \eta^{2}=0.54\right)$. Bonferroni corrected pairwise comparisons pointed out a significant difference between the hMT+-rTMS (experimental site) and both the PPC-rTMS (control site) and the NO TMS condition, but not a significant difference between the NO TMS condition and the PPC-rTMS condition (Fig. 3). In addition, we found a significant effect of the factor Speed Gap $\left(F_{4,52}=321.87\right.$, $\left.p<0.05, \eta^{2}=0.96\right)$; Bonferroni corrected pairwise comparisons revealed significant differences between all the Speed Gaps. In particular, we obtained, on average, 2.08, $16.81,58.18,84.23$ and $93.75 \%$ of responses reporting a speed change for Speed Gaps of 0, 1, 2, 3 and 4 respectively (SE: 0.85, 2.6, 5.2, 2.94 and 1.61, respectively). Moreover, the ANOVA reported a significant interaction between TMS and Speed Gap $\left(F_{8,104}=2.91, p<0.01\right.$, $\left.\eta^{2}=0.18\right)$. Bonferroni corrected pairwise comparisons reported for the Speed Gap $=1$ a significant difference

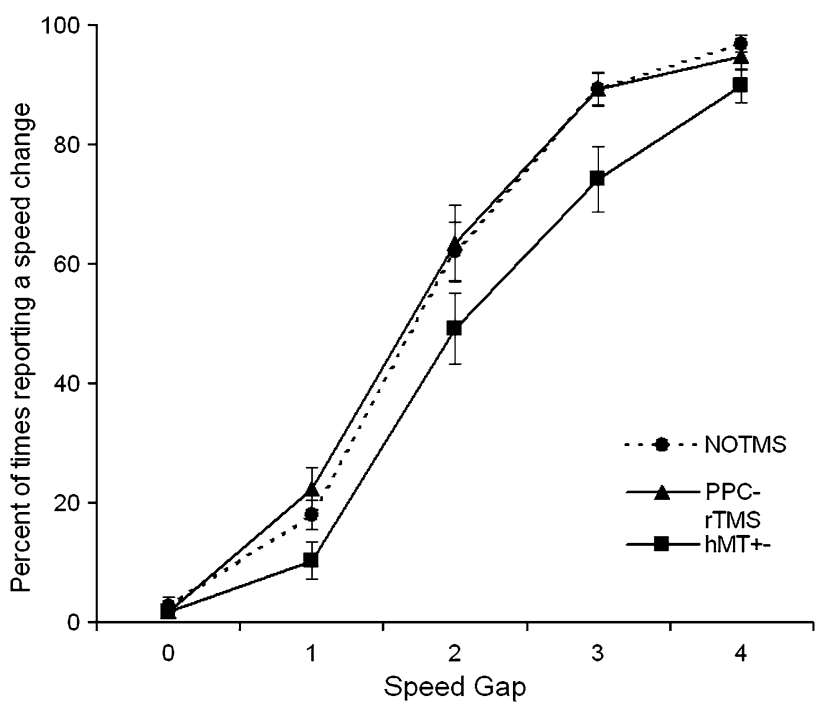

Fig. 3 Results of the main experiment. The percentage of times reporting a speed change is plotted as a function of the speed gaps for the hMT+-rTMS condition, PPC-rTMS condition, and the No-rTMS control condition. The abscissa shows the speed gaps whereas the ordinate the percentage of responses reporting a speed change. Curves are reported for the No-rTMS condition, PPC-rTMS, and hMT+rTMS. Since the main effect of speed direction and the interaction between TMS and speed direction were not significant, the two speed directions were collapsed. Error bars \pm SEM 
Fig. 4 Results of the main experiment. Mean thresholds (a) and mean slopes (b) for the no-rTMS condition, PPC-rTMS, and hMT+-rTMS. Error bars \pm SEM
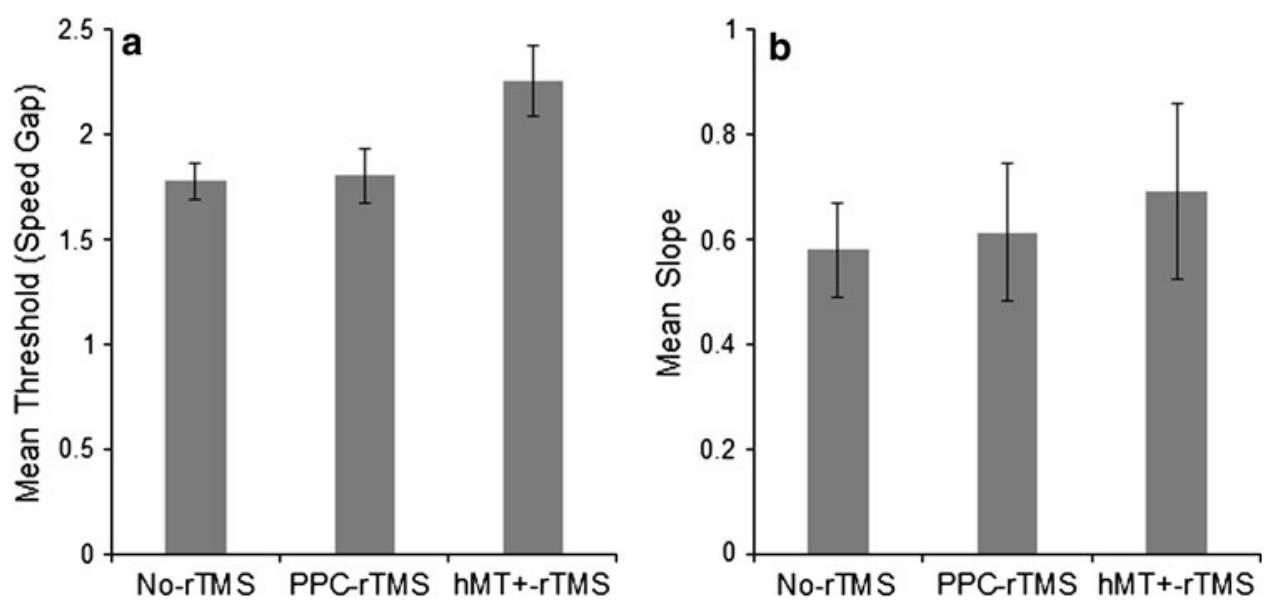

between the hMT+-rTMS condition and both the PPCrTMS and the NO TMS conditions, but not a significant difference between the NO TMS and the PPC-rTMS condition. For the other Speed Gaps the corrected pairwise comparisons reported only a significant difference between the hMT+-rTMS condition and the PPC-rTMS condition.

As the interval between the speed change and the TMS onset was constant, participants could have learnt this association, leading them to respond after the TMS pulses. To test this possibility, we performed a repeated measures ANOVA, including the Number of Responses as a factor in the three TMS conditions, collapsing the number of responses across all the speed gaps (i.e. No-rTMS: mean $=43.0, \quad \mathrm{SE}=1.3 ; \quad$ PPC-rTMS: $\quad$ mean $=43.4$, $\mathrm{SE}=2.0 ;$ hMT+-rTMS: mean $=36.0, \mathrm{SE}=2.5$ ). The repeated measures ANOVA showed a significant effect of rTMS $\left(F_{2,26}=15.41, p<0.01, \eta^{2}=0.54\right)$. Bonferroni corrected pairwise comparisons revealed that, in the case of the hMT+-rTMS condition the number of responses was significantly lower than in the No-rTMS and PPC-rTMS conditions. Moreover, there was no a significant difference between the No-rTMS and PPC-rTMS conditions. These results clearly indicate that participants were not using rTMS as a response cue.

To further establish the effectiveness of the TMS stimulation, we fitted a logistic function (see Eq. 1) to the percentage of times a speed change was reported. In particular, we considered the $50 \%$ point of the logistic function (midpoint) in order to obtain a measure of the effect of rTMS on the detection of speed changes when applied over hMT+ with respect to the other control conditions (i.e. PPC-rTMS and NO TMS). In this case, the threshold values express the difference between two speeds that can be detected with a probability of 0.5 . For clarity, we subsequently converted the speed gap threshold values into the difference between the two speeds (in $\mathrm{cm} / \mathrm{s}$ ). Moreover, the goodness of the resulting fit was quantified by calculating the adjusted $R$-squared.
There was an overall increase of the thresholds for the hMT+-rTMS, indicating that, in this condition, subjects needed a larger speed gap to detect the change (see Fig. 4a). Since a repeated measures ANOVA did not report a significant effect of Speed Direction $\left(F_{1,13}=0.90\right.$, $\left.p>0.05, \eta^{2}=0.06\right)$ nor a significant interaction between TMS and Speed Direction factors $\left(F_{2,26}=0.79, p>0.05\right.$, $\left.\eta^{2}=0.05\right)$, we recalculated the thresholds collapsing the two directions (i.e. descending and ascending).

A repeated-measures ANOVA on these thresholds indicated a significant effect of the main factor TMS $\left(F_{2,26}=13.29, p<0.05, \eta^{2}=0.50\right)$; Bonferroni corrected pairwise comparisons revealed a significant difference between the mean threshold estimated for the hMT+rTMS (mean: 2.26; SE: 0.16), in comparison to both the PPC-rTMS (mean: 1.80; SE: 0.13) and No-rTMS conditions (mean: 1.78 SE: 0.09). No significant difference was found between the threshold estimated in the PPC-rTMS and No-rTMS conditions. Moreover, the adjusted $R$ squares estimated were 0.99 for all the TMS conditions (SE: $0.002,0.005,0.001$ for No-rTMS, PPC-rTMS and hMT+-rTMS, respectively), confirming that the logistic function (Eq. 1) provided a good account for our data.

Subsequently, the values for speed gap threshold were converted in terms of speed difference that can be detected with a probability of 0.5 . We calculated the mean of the differences between speeds, relative to each speed gap, thus obtaining a series of values that were fitted with a linear function $\left(Y=0.9506 \times X+0.0308 ; R^{2}=0.99\right)$. The resulting thresholds for detecting a speed change correspond to speed differences of $1.72,1.75$ and $2.18 \mathrm{~cm} / \mathrm{s}$ for the No-rTMS, PPC-rTMS and hMT+-rTMS conditions, respectively. In addition, a repeated-measures ANOVA performed on the slopes of the best-fitting psychometric functions (Fig. 4b) did not reveal significant effect for the main factor TMS $\left(F_{2,26}=1.63, p>0.05, \eta^{2}=0.11\right)$.

The False Alarm probability of the psychometric functions based on the collapsed data were also analysed: and 
its estimation resulted to be very low across all the TMS conditions (i.e. No-rTMS: 0.023, SE: 0.021; PPC-rTMS: 0.013, SE: 0.01; hMT+-rTMS: 0.006, SE: 0.006). This result suggests that participants had a relatively conservative criterion in reporting a change in speed. A repeated measures ANOVA on the False Alarms probability did not report any significant effect of TMS $\left(F_{2,26}=1.51\right.$, $\left.p>0.05, \eta^{2}=0.10\right)$, indicating no difference between the three conditions.

\section{Discussion}

The present experiment showed that, in the hMT+-rTMS condition, the detection of speed changes was significantly affected. The overall increase of the threshold when hMT+ was stimulated indicates that participants required a larger difference between consecutive speeds (i.e. speed gap) to detect a change, in comparison to both control conditions. This change in performance after hMT+-rTMS suggests a clear involvement of this region in detecting speed changes with tactile moving stimuli.

Moreover, the absence of a significant difference between the slopes of the psychometric functions indicated that there were no differences in subjects' ability to discriminate changes in speed (according to Mather and Pavan 2009).

\section{General Discussion}

Despite the increasing number of studies that have shown involvement of the extrastriate $\mathrm{hMT}+$ region in tactile motion perception, some important methodological concerns still make this hypothesis a matter for debate. Critically, most of the previous studies used correlational methods such as fMRI (Hagen et al. 2002; Ricciardi et al. 2007; Beauchamp et al. 2007; Matteau et al. 2010), which prevents direct investigation of the causal role of this area in the motion detection network. Only our previous study (Ricciardi et al. 2011) has used TMS to support the hypothesis that the hMT+ region encodes not only visual signals, but also information from other sensorial modalities. However, some methodological criticisms have led to a lack of faith in these results. That said, the methodology and experimental design used in the present study produced stable data, in turn, providing strong support for the proposed involvement of $\mathrm{hMT}+$ in tactile motion processing. In fact, this study did not aim to simply replicate the result of Ricciardi et al. (2011), but also to address the experimental limitations mentioned above. Thus, we aimed to resolve them by elaborating a psychophysical analysis and including an additional control condition to evaluate important characteristics of the perceptual processing more effectively. Overall, the results provided confirmation for the conditions used in previous TMS study: among the conditions evaluated, the one we selected (i.e. the larger cylinder, along with its set of speeds), indeed produced the most robust performance in the speed detection task. Although the angular speed was the same among the cylinders, their diameter was directly proportional to both the tangential speeds and the range. The manipulation of these variables in the preliminary experiment led to a very different pattern of results in terms of percentage of times reporting a speed change. In particular, we found that the experimental speeds in the range of $16-20 \mathrm{~cm} / \mathrm{s}$ were the most suitable parameters to produce a high percentage of times reporting a speed change. However, the values obtained from the discarded cylinders suggest that the manipulation of a lower set of speeds (e.g. 12-16 cm/s) could be potentially intriguing for a future study, aimed at determining more precisely the lower bounds of the tactile speed perception process.

In the main experiment, participants were presented with and without TMS stimulation in within-subject conditions, which allowed quantification of the impact of TMS stimulation. However, one cannot quantify the TMS effect without a reference point, such as a performance free of TMS pulses. In fact, it could be the case that the stimulation of the PPC enhanced performance, while hMT+ stimulation produced a null effect. The performance in the no-rTMS condition was, however, comparable to the performance during PPC-rTMS condition, thus suggesting that TMS applied to the hMT+ did actually influence speed perception processing. The introduction of a further control condition was necessary in order to determine the absolute impact of the TMS stimulation, which was a limitation in the previous work (Ricciardi et al. 2011).

One of the main motivations for the current study was to determine whether psychometric functions for the detection of speed changes conformed to the prediction of higher thresholds and shallower slopes when rTMS was applied over hMT + . That is, if rTMS over hMT + produced a shallower slope of the psychometric function than in the other conditions, while maintaining the threshold constant (i.e. in respect of the other TMS conditions), this should have supported the presence of a greater internal noise. In turn, this would have affected speed change detection and response times, as pointed out by Ricciardi et al. (2011). However, this was not the case: the absence of a difference between slopes and the presence of a significant difference between the detection thresholds seem to indicate that $\mathrm{hMT}+$ is truly involved in tactile motion perception. Indeed, rTMS affected the process underlying speed encoding and did not merely introduce greater internal noise (Buss et al. 2009). Nevertheless, it is unlikely that 
motion processing would be related to only visuo-tactile modalities, and not the management of the motion process per se, as several studies have already suggested (Wolbers et al. 2010; Bedny et al. 2010). The task used in this study involved the detection of speed changes; a mechanism considered a distinct aspect of motion processing (see Khuu and Badcock 2002). Nonetheless, such a distinction has been criticised by van Boxtel et al. (2006), whose findings clearly supported a unified model of speed and motion perception. One might query the paradigm used in that study, by noting that participants responded to a frequency change in tactile perception, rather than to speed processing itself, and that a simple motion/non-motion task may have been more suitable. However, the finger surface in contact with the cylinders was around $1 \mathrm{~cm}^{2}$, and the tactile stimulus dots were translated onto this surface. Thus, since such translation is perceived as movement, it would not be reasonable to expect that participants represented the stimulation as a simple change in frequency of dots. Moreover, given that the information needed to determine the occurrence of either acceleration or deceleration is based on a more subtle distinction than that required for a motion/non-motion task (and that motion would be perceived in any event), the conclusions drawn from the speed change detection here may definitively be extended to motion perception. To this end there is psychophysical evidence that tactile motion perception depends strictly on the temporal frequency of the moving surface. Dépeault et al. (2008), for example, found a monotonic relation between subjective speed and the temporal frequency of a moving dot surface. Such dependency suggests that tactile and visual moving stimuli are processed similarly at the sensory level. Moreover, Bensmaïa et al. (2006) found that when a tactile grating drifted in the same direction as that of a distractor visual grating (presented simultaneously), the visual distractor increased the perceived speed of the tactile grating. This effect was proportional to the temporal frequency, rather than the perceived speed of the gratings, suggesting that the visualtactile interaction depends on motion and not simply the oscillations inherent in drifting sinusoids. On the other hand, further studies are necessary to clarify the role of the temporal frequency in tactile motion perception. This would serve to investigate more deeply whether tactile and visual motion processing share not only the same neural substrates, but also the same neural populations with specific spatiotemporal tuning.

\section{Conclusions}

Our findings strengthen the evidence that recruitment of the $\mathrm{hMT}+$ is necessary for tactile motion processing and show a causal role of this area in non-visual sensory motion processing in human subjects. Many limitations of the Ricciardi et al.'s (2011) study were resolved here, while some others may still need to be explored further. Future work will be required to pursue a higher level of specification, by investigating the role of the anterior and posterior hMT + regions and how these regions interact in managing the different aspects of cross-modal representations (i.e. by including both visual and tactile modalities in the same experimental context). An approach involving TMS co-registration with EEG or fMRI is highly encouraged, in order to determine whether tactile and visual modalities share the same anatomical and functional circuitry.

Acknowledgments This research was supported by the European Union (grant IST-2006-027141 to P.P.), and by the Italian Ministero dell'Istruzione, dell'Università e della Ricerca (PRIN 2006117208 to P.P. and T.V.; RBNE018ET9-003 to P.P.). Authors also thank Dr. Elisabeth Blagrove for her help on language revision.

\section{References}

Bar M (2007) The proactive brain: using analogies and associations to generate predictions. Trends Cogn Sci 11:280-289

Beauchamp MS (2005) See me, hear me, touch me: multisensory integration in lateral occipital-temporal cortex. Curr Opin Neurobiol 15(2):145-153

Beauchamp MS, Yaser NE, Kishan N, Ro T (2007) Human MST but not MT responds to tactile stimulation. J Neurosci 27:8261-8267

Bedny M, Konkle T, Pelphrey K, Saxe R, Pascual-Leone A (2010) Sensitive period for a multi-modal response in human MT/MST. Curr Biol 20(21):1900-1906

Bensmaïa SJ, Killebrew H, Craig JC (2006) Influence of visual motion on tactile motion perception. J Neurophysiol 96:1625-1637

Bicchi A, Scilingo EP, Ricciardi E, Pietrini P (2008) Tactile flow explains haptic counterparts of common visual illusions. Brain Res Bull 75:737-741

Blake R, Sobel KV, James TW (2004) Neural synergy between kinetic vision and touch. Psychol Sci 15:397-402

Born RT, Bradley DC (2005) Structure and function of visual area MT. Ann Rev Neurosci 28:157-189

Buss E, Hall JW, Grose JH (2009) Psychometric functions for pure tone intensity discrimination: slope differences in school-aged children and adults. J Acoust Soc Am 15:1050-1058

Dépeault A, Meftah E, Chapman CE (2008) Tactile speed scaling: contributions of time and space. J Neurophysiol 99:1422-1434

Ganis G, Schendan HE (2008) Visual mental imagery and perception produce opposite adaptation effects on early brain potentials. Neuroimage 42:1714-1727

Gleich O, Hamann I, Kittel MC, Klump GM, Strutz J (2006) A quantitative analysis of psychometric functions for different auditory tasks in gerbils. Hear Res 220:27-37

Hagen MC, Franzen O, McGlone F, Essick G, Dancer C, Pardo JV (2002) Tactile motion activates the human middle temporal/V5 (MT/V5) complex. Eur J Neurosci 16:957-964

Harris JA, Clifford CW, Miniussi C (2008) The functional effect of transcranial magnetic stimulation: signal suppression or neural noise generation? J Cogn Neurosci 20:734-740

Khuu SK, Badcock DR (2002) Global speed processing: evidence for local averaging within, but not across two speed ranges. Vis Res 42:3031-3042 
Konkle T, Wang Q, Hayward V, Moore CI (2009) Motion aftereffects transfer between touch and vision. Curr Biol 19:1-6

Kosslyn SM (1994) Image and brain. MIT Press, Cambridge

Kosslyn SM, Ganis G, Thompson WL (2001) Neural foundations of imagery. Nature Rev Neurosci 2:635-642

Lacey S, Tal N, Amedi A, Sathian K (2009) A putative model of multisensory object representation. Brain Topogr 21:269-274

Lacey L, Flueckiger P, Stilla R, Lava M, Sathian K (2010) Object familiarity modulates the relationship between visual object imagery and haptic shape perception. NeuroImage 49:1977-1990

Lam CF, Mills JH, Dubno JR (1996) Placement of observations for efficient estimation of a psychometric function. J Acoust Soc Am 99:3689-3693

Mather G, Pavan A (2009) Motion-induced position shifts occur after motion integration. Vision Res 49:2741-2746

Mather G, Pavan A, Campana G, Casco C (2008) The motion aftereffect reloaded. Trends Cogn Sci 12:481-487

Matteau I, Kupers R, Ricciardi E, Pietrini P, Ptito M (2010) Beyond visual, aural and haptic movement perception: hMT+ is activated by electrotactile motion stimulation of the tongue in sighted and in congenitally blind individuals. Brain Res Bull 82(5-6):264-270

McKeefry DJ, Burton MP, Vakrou C, Barrett BT, Morland AB (2008) Induced deficits in speed perception by transcranial magnetic stimulation of human cortical areas V5/MT+ and V3A. J Neurosci 28:6848-6857

Miniussi C, Ruzzoli M, Walsh V (2010) The mechanism of transcranial magnetic stimulation in cognition. Cortex 46:128-130

Oldfield RC (1971) The assessment and analysis of handedness: the Edinburgh inventory. Neuropsychologia 9:97-113

Pridmore S, Fernandes Filho JA, Nahas Z, Liberatos C, George MS (1998) Motor threshold in transcranial magnetic stimulation: a comparison of a neurophysiological method and a visualization of movement method. J ECT 14:25-27

Ptito M, Schneider FC, Paulson OB, Kupers R (2008) Alterations of the visual pathways in congenital blindness. Exp Brain Res 187:41-49

Ricciardi E, Bonino D, Gentili C, Sani L, Pietrini P, Vecchi T (2006) Neural correlates of spatial working memory in humans: a functional magnetic resonance imaging study comparing visual and tactile processes. Neuroscience 139:339-349
Ricciardi E, Vanello N, Sani L, Gentili C, Scilingo EP, Landini L, Guazzelli M, Bicchi A, Haxby JV, Pietrini P (2007) The effect of visual experience on the development of functional architecture in hMT+. Cereb Cortex 17:2933-2939

Ricciardi E, Basso D, Sani L, Bonino D, Vecchi T, Pietrini P, Miniussi C (2011) Functional inhibition of the human middle temporal cortex affects non-visual motion perception: a repetitive transcranial magnetic stimulation study during tactile speed discrimination. Exp Biol Med 236:138-144

Ruzzoli M, Marzi CA, Miniussi C (2010) The neural mechanisms of the effects of transcranial magnetic stimulation on perception. J Neurophysiol 103:2982-2989

Sack AT, Kohler A, Linden DE, Goebel R, Muckli L (2006) The temporal characteristics of motion processing in hMT/V5+: combining fMRI and neuronavigated TMS. NeuroImage 29:1326-1335

Rossi S, Hallett M, Rossini PM, Pascual-Leone A, Safety of TMS Consensus Group (2009) Safety, ethical considerations, and application guidelines for the use of transcranial magnetic stimulation in clinical practice and research. Clin Neurophysiol 120:2008-2039

Théoret H, Kobayashi M, Ganis G, Di Capua P, Pascual-Leone A (2002) Repetitive transcranial magnetic stimulation of human area MT/V5 disrupts perception and storage of the motion aftereffect. Neuropsychologia 40:2280-2287

Tootell RB, Reppas JB, Dale AM, Look RB, Sereno MI, Malach R, Brady TJ, Rosen BR (1995) Visual motion aftereffect in human cortical area MT revealed by functional magnetic resonance imaging. Nature 375:139-141

van Boxtel JJA, van Ee R, Erkelens CJ (2006) A single system explains human speed perception. J Cogn Neurosci 18:1808-1819

Van Orden GC, Paap KR (1997) Functional neuroimages fail to discover pieces of mind in the parts of the brain. Philos Sci 64:S85-S94

Walsh V, Pascual-Leone A (2003) Transcranial magnetic stimulation: a neurochronometrics of mind. MIT Press, Cambridge

Wolbers T, Zahorik P, Giudice NA (2010) Decoding the direction of auditory motion in blind humans. NeuroImage 56(2):681-687 